\title{
Highlights at ISOLDE
}

\author{
K. Blaum ${ }^{1}$, M. J. G. Borge ${ }^{2,3}$, B. Jonson ${ }^{4}$ and P. Van Duppen ${ }^{5}$ \\ ${ }^{1}$ Max-Planck-Institut für Kernphysik, D-69117 Heidelberg, Germany \\ ${ }^{2}$ ISOLDE-PH, CERN, CH-1211 Geneva-23, Switzerland \\ ${ }^{3}$ Instituto de Estructura de la Materia, CSIC, Serrano 113 bis, \\ E-28006 Madrid, Spain \\ ${ }^{4}$ Fundamental Physics, Chalmers University of Technology, \\ SE-41296 Göteborg, Sweden \\ ${ }^{5}$ KU Leuven, Instituut voor Kern- en Stralingsfysica, \\ B-3001 Leuven, Belgium \\ 2mgb@cern.ch
}

\begin{abstract}
The ISOLDE Radioactive Ion Beam Facility at CERN started fifty years ago as an interesting attempt to widen the palette of nuclear species for experimental investigations. During this half century, one has witnessed a continuous development and refinement of the experimental programme. On the road towards today's installation many scientific breakthroughs have been achieved. We present some of them here.
\end{abstract}

\section{Introduction}

The ISOLDE Radioactive Beam Facility is the dedicated CERN installation for the production and acceleration of radioactive nuclei. Isotopes from a variety of elements are produced in a target directly connected to the ion source of an isotope separator, which results in a very short time-delay between production of a nucleus and its arrival at the experimental set-up. Thus, the possibility to study isotopes with extreme neutron-to-proton ratios and with very short half-life, is provided. The radioactive isotopes produced at ISOLDE are used in experiments in nuclear-, atomic-, solid-state- and biophysics, as well as in applications, particularly in medicine. The study of properties of nuclei all over the nuclear landscape gives not only clues to a detailed understanding of the structure of the nucleus but also about reactions in the Cosmos, where the chemical elements building up the Nature around us are born (Fig. 1).

The pioneering experiment using an isotope separator directly linked to an accelerator was carried out in Copenhagen already in $1951 .^{1}$ Inspired by this achievement, the European nuclear-physics community proposed to build a generalpurpose experiment for the production of short-lived isotopes connected to the synchrocyclotron (SC) at CERN. The project was approved on December 17, 1964 by the CERN director Victor Weisskopf. An underground laboratory was built and 


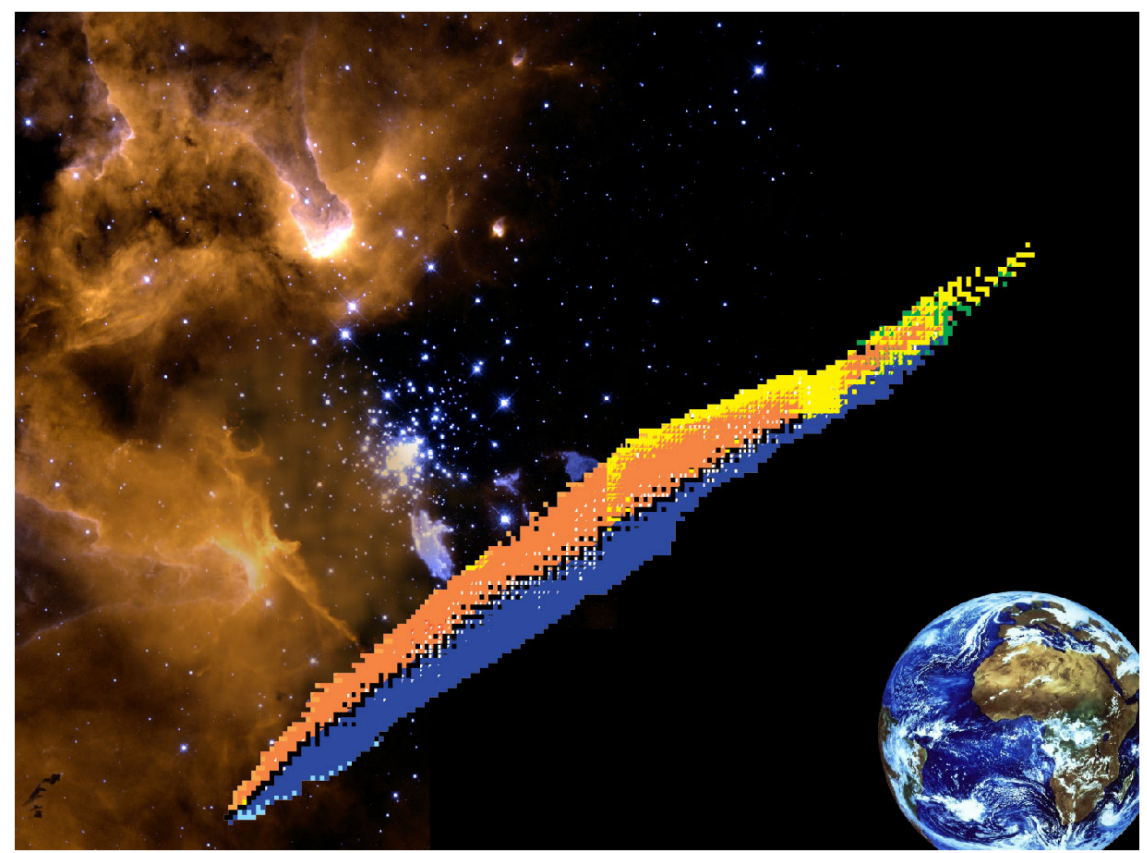

Fig. 1. Atomic nuclei are organised in a grid of squares, each of which represents a certain number of protons (vertically) and neutrons (horizontally), together forming the chart of nuclides, as shown here. The black squares are the stable nuclei and indicate the valley of stability. This chart, or nuclear landscape, is the working field of ISOLDE, where the main emphasis is on the most exotic nuclei. The nuclei studied at ISOLDE give important new insight in the complex nuclear manybody system. They give clues to the simplicity hidden in the complexity, they tell us about the elements that build up the Nature around us and about their cosmic origin and plays a prominent role in our understanding of the formation of the chemical elements.

protons from the SC were brought via a tunnel to hit a production target. The first experiment at this on-line isotope separator, named ISOLDE, was performed on September 17, 1967 (Fig. 2). ISOL (acronym for Isotope Separator On Line) has since then been the standard name for this type of radioactive isotope production method - the ISOL technique.

Just at the time of the first successful experiments at the new underground hall, CERN decided for a major upgrade of the SC. This SC improvement programme (SCIP) aimed at an increase of the internal beam intensity from 1 to $10 \mu \mathrm{A}$ together with an improved extraction efficiency giving a proton beam intensity increase of more than a factor 100 at the ISOLDE target. An essential part of the upgrade was to change the frequency system at the SC, which had been based on a tuning fork, to a rotating condenser. In order to cope with the higher proton current offered to ISOLDE, an advanced technical development programme was launched. A new design of the target-ion source systems was proposed and, as it turned out, gave access to more and more isotopes of different chemical elements. The SCIP 


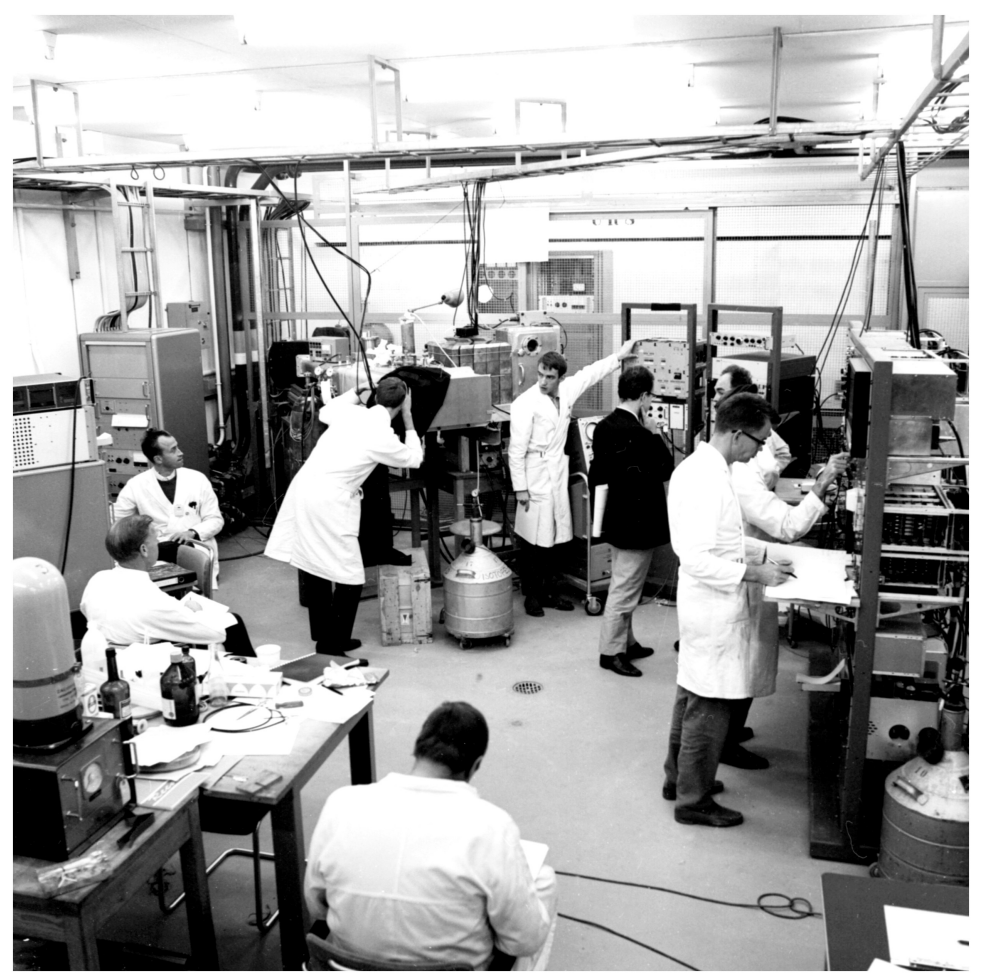

Fig. 2. The ISOLDE experimental hall in 1967. Note that ISOLDE at that time was part of the CERN Nuclear Chemistry Group, which meant white lab coats.

programme took place in the years 1972-1974 and the new layout of the separator and its target-ion source became referred to as ISOLDE 2. The high intensity of produced isotopes and the large variety of different elements meant that ISOLDE had become a major international facility to perform experiments on radioactive isotopes.

The SC machine had been in operation since 1957 and it became clear in the middle of the 1980s that this accelerator had to be closed. To maximise the use of the last years of the SC, the ISOLDE Collaboration proposed to build a second isotope separator. This new separator, ISOLDE 3, was constructed with a two-stage separation (one $90^{\circ}$ magnet followed by a $60^{\circ}$ one) to achieve a very high mass resolution. A new target was placed in the $\mathrm{SC}$ vault and the produced radioactive isotopes were brought into the proton hall. The new separator gave a mass resolution of $\mathrm{M} / \Delta \mathrm{M}$ of 7000 and was a pre-runner for the design of the present High-Resolution Separator (HRS) at the PS-Booster.

The future of the ISOLDE programme after the SC shutdown was discussed and the general consensus was that the most attractive option would be to move ISOLDE closer to the PS complex and to place its targets in an extracted $1 \mathrm{GeV}$ proton beam 
from the PS Booster. The ISOLDE Collaboration set up a Technical Committee that helped CERN to find the optimal design of the new facility. A suitable layout was found and on May 4, 1990 the CERN Directorate approved the proposal to move ISOLDE to the PS Booster. The ground work for the new ISOLDE building started in October. At noon on December 19, 1990 the last shift of protons was delivered to ISOLDE from the SC leaving a legacy of more than a quarter of a century of pioneering experiments that benchmarked the future of the ISOL facilities in the world. The ISOLDE-PS Booster Facility was built in the usual CERN spirit and already in May 1992 the new installation could be inaugurated. The first experiment, a study of the beta decay of the two-proton halo nucleus ${ }^{17} \mathrm{Ne},{ }^{2}$ was successfully completed on June 26.

The ISOLDE programme was traditionally mainly dedicated to study nuclear ground-state properties and excited nuclear states populated in radioactive decays. With the large palette of different isotopes, some of them produced with high intensity, it was an attractive possibility to build a post-accelerator at ISOLDE. In 1994, such a proposal was presented to CERN asking for permission of the ISOLDE community to build a suitable accelerator to get exotic nuclear beams in the energy range of $2-3 \mathrm{MeV} / \mathrm{u}$. The project was approved and the REX-ISOLDE accelerator was built in an extension to the experimental hall (see Fig. 3 and next section for details). The first beams were accelerated on October 31, 2001, and this addition to the ISOLDE programme has turned out to be both successful and very prolific.

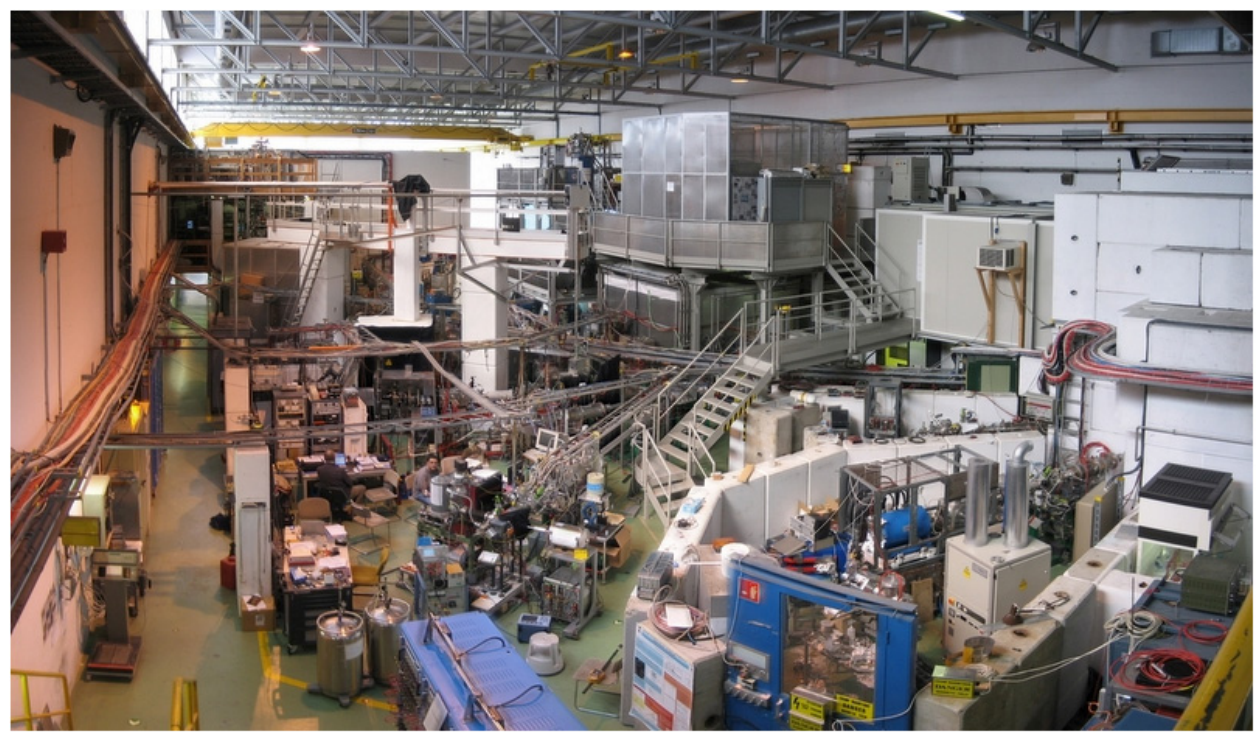

Fig. 3. The ISOLDE experimental hall in 2007. 


\section{Production, Manipulation and Acceleration of Radioactive Ion Beams}

The success of the ISOLDE facility is based on intertwined developments of radioactive ion beams (RIB) and instrumentation for physics experiments. The cross fertilisation leads to a broad spectrum of beams available with masses varying from ${ }^{6} \mathrm{He}$ to ${ }^{232} \mathrm{Ra}$, with half-lives down to the $\mathrm{ms}$ range (e.g. ${ }^{14} \mathrm{Be}$ $\mathrm{T}_{1 / 2}=4.45 \mathrm{~ms}$ ), intensities up to the $\mathrm{nA}$ level (e.g. ${ }^{213} \mathrm{Fr}$ with $\sim 8 \times 10^{9}$ particles per second) and energies from rest to a few $\mathrm{MeV} / \mathrm{u} \cdot{ }^{3,}{ }^{4} \mathrm{~A}$ continuous development programme implementing new techniques, like e.g. the use of nano-structured target material, laser resonance ionisation, ion cooling and charge state breeding, keeps the facility at the forefront of RIB science ever since it was constructed. The RIB production and manipulation process adapts the beam properties to the different experimental setups. As one mainly deals with short-lived radioactive isotopes that are produced in minute quantities compared to the vast amount of unwanted species produced (ratios over $10^{12}$ between the production rate of the unwanted versus wanted isotopes are routinely reached) the overall RIB production process has to be efficient, fast and selective.

\subsection{The target-ion source system - The heart of the matter}

ISOLDE's radioactive isotopes are produced in high-energy proton induced reactions impinging on different target materials. The primary proton beams from the CERN-PS Booster induce spallation, fragmentation and fission reactions which allow, by a proper choice of the target material, to produce a range of isotopes that covers a substantial part, $80 \%$, of the chart of nuclei below uranium $(Z=92)$. As the reaction mechanisms are barely selective, the target-ion source system at the origin of the low-energy ion beam combined with the mass analysing magnet and other ion manipulation devices are used to reduce the unwanted contaminants and/or to identify the isotopes of interest. Pioneering work was necessary to integrate the target and the ion source into one compact system that is kept at high temperature to speed up the diffusion and effusion of the radioactive atoms from the target container. ${ }^{3}$ This led to a successful design that today is still competitive and that allows using different atomic and chemical processes to purify the beam. A simple but effective approach is cooling the transfer line between target and ion source, allowing only the gaseous elements (noble gases) or most volatile molecules to reach the ion source. The suppression of elements that make a chemical bonding with the surface of a quartz line installed between target and ion source represents another approach. Recently, new developments including the use of nano-structured target materials are explored to reduce the delay time and obtain more ruggedised systems. 
ISOLDE's successful laser spectroscopy programme and the fact that powerful pulsed laser systems became available led to the implementation of laser resonance ionisation for the production of RIB in the mid-1980s. ${ }^{5}$ This element selective and efficient ionisation process, that is based on the use of different laser beams to invoke multi-step atomic excitations into the continuum, results in clean beams. The first on-line production of photo-ionised radioactive $\mathrm{Yb}$ beams was soon followed by isotopes from a wide range of different elements. ${ }^{6,7}$ Now the laser ion source is routinely used for over $50 \%$ of ISOLDE's beam time. A recent improvement of the selectivity of the laser ionisation is the Laser Ion Source Trap (LIST) ${ }^{8}$ approach that integrates a standard target-ion source system, laser ionisation and ion manipulation. It is based on the photo-ionisation of the plume of atoms escaping from the high temperature ISOLDE target-ion source system, subsequent capturing of the ions in a radio-frequency trap and transporting them to the extraction region. While losses in overall efficiencies are encountered, LIST improves the selectivity by about four orders of magnitude. ${ }^{9}$

\subsection{Cooled beams, isomeric beams and in-source laser spectroscopy}

Adapting the longitudinal and transverse RIB emittance or the beam pulse characteristics to the needs of the experiments was pioneered at ISOLDE's highprecision mass spectrometry set-up ISOLTRAP. The potential of buffer-gas cooling in radio-frequency or in Penning traps to produce cooled, bunched radioactive ion beams with good efficiency could be demonstrated. Larger versions of both the radio-frequency quadrupole ion trap and of the Penning trap were developed to deliver cooled and bunched beams to other ISOLDE users like the collinear laser spectroscopy set-up (see Section 5) where it increased the signal-to-background ratio up to four orders of magnitude, and to the REX-ISOLDE post-accelerator (see Section 2.3).

Soon after the first laser ionised RIB, beams of long-lived states, called isomeric, were produced and separated using the hyperfine splitting of the atomic transition ${ }^{11}$ as it depends on the nuclear properties of the isomer. By changing the laser frequency of the first atomic transition, the specific hyperfine structure of the different nuclear states can be probed. Combining this isomer selectivity with $\beta$-decay and mass spectroscopy studies led to the discovery of three $\beta$-decaying states in ${ }^{70} \mathrm{Cu}$ : the ground state and two isomeric states (see Fig. 4). Their existence could be explained as due to the coupling of one proton and one neutron to a ${ }^{68} \mathrm{Ni}$ core. ${ }^{12}$ With ISOLDE's post-accelerator (see Section 2.3 ), isomeric beams were postaccelerated and used for Coulomb excitation measurements probing the strength of the $Z=28$ shell and $N=40$ sub-shell closures. ${ }^{13}$ This pioneering experiment moreover demonstrated that Coulomb excitation could trigger the depopulation of 

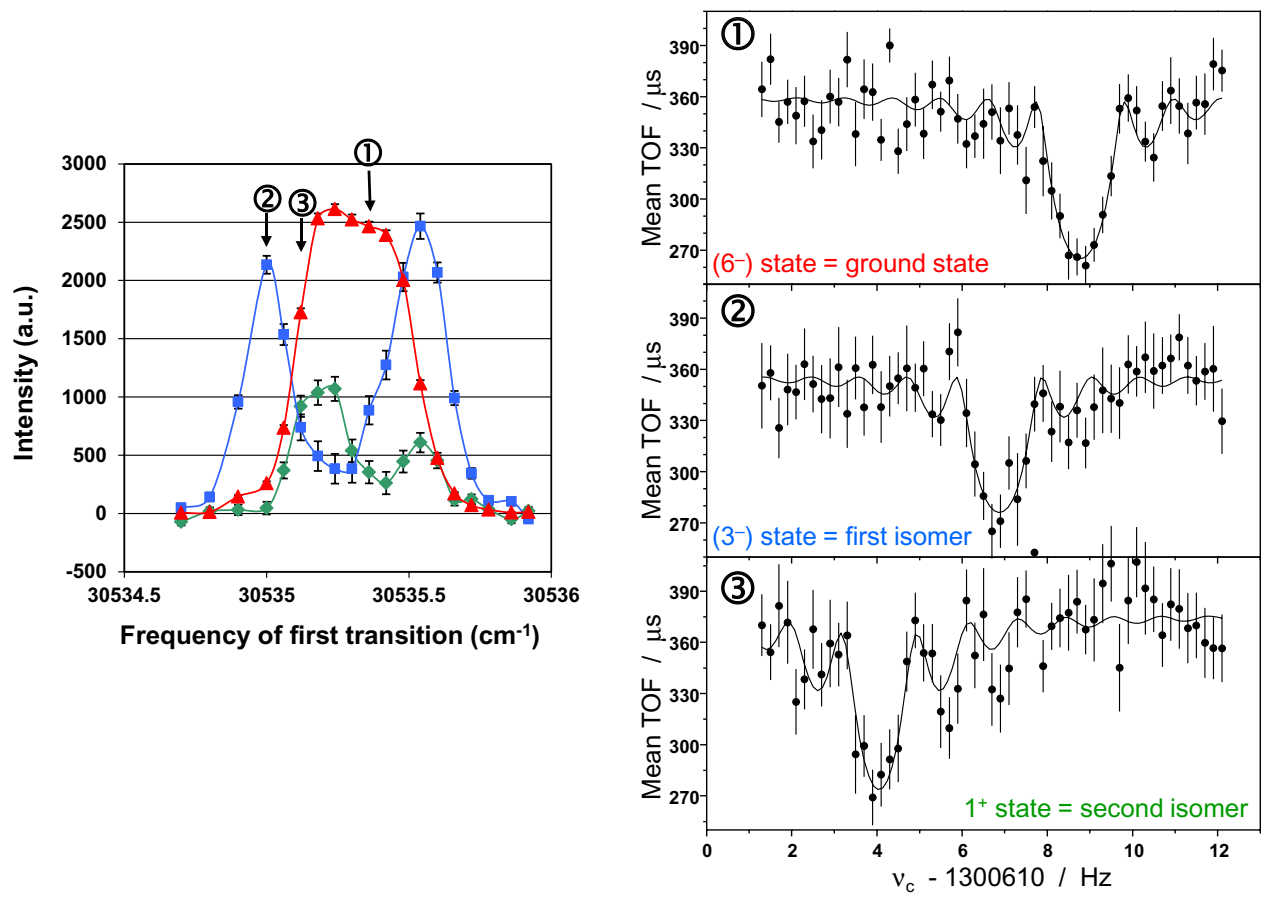

Fig. 4. Isomer selection is performed using the hyperfine splitting of the atomic levels probed in the resonant laser ionisation process used in the ISOLDE laser ion source. The yield of the different isomers in ${ }^{70} \mathrm{Cu}$ as a function of the laser frequency of the first atomic transition are shown for the ground state $\left(6^{-}\right)$(triangles), $\left(3^{-}\right)$(squares) and $1^{+}$(circles) $\beta$-decaying isomers of ${ }^{70} \mathrm{Cu}$ (left). The modified cyclotron frequency resonance spectra obtained at ISOLTRAP are shown for three different laser frequencies $(1,2$ and 3$)$. The line represents a fit through the data and the resonance frequency is inversely proportional to the mass of the nuclear state. The ISOLTRAP measurements demonstrated the presence of three different long-lived states in ${ }^{70} \mathrm{Cu}$ and to obtain their mass whose difference is in perfect agreement with $\beta$-spectroscopy studies (right). The line is a fit through the data points. The spectrum on the bottom right (3) was obtained after an extra purification step in the Penning trap.

an isomer towards an excited state that subsequently decays to the ground state, which called for a detailed study of other spin-multiplets in odd-odd nuclei.

Because of the high sensitivity of the laser ion source, laser ionisation spectroscopy measurements became possible with very weak beams (intensities down to less than one atom per second). However, this so-called in-source laser spectroscopy method was mainly limited to heavy mass nuclei because of the limited spectral resolution of the method. Charge radii and electromagnetic moments of a number of neutron deficient nuclei around the lead isotopes $(Z=82)$ were obtained extending the pioneering work on optical spectroscopy of the mercury isotopes using samples from ISOLDE $^{14}$ (see Section 4). This technique allowed for the determination of the unknown ionisation potential of astatine, the only element in the table of Mendeleev below uranium for which this fundamental atomic property was not 
known experimentally. ${ }^{15}$ The result benchmarks quantum chemistry calculations and has moreover an impact on the field of innovative medical radioisotope production. For example the isotope ${ }^{211} \mathrm{At}$, because of its decay properties, is an interesting pharmaceutical radioisotope for targeted alpha therapy in cancer treatment provided its chemistry is well understood.

The large variety of RIBs with different decay properties and from various elements make them tailored probes for condensed matter and biophysics studies. The radioactive atoms act as spies and their emitted radiation provides information on their lattice position or on the magnetic and electrical properties of the surrounding atoms. Because of the high radiation detection efficiency, only very low concentrations of radioactive impurity atoms are necessary to provide unique nano-scale information in materials, surfaces or interfaces (see Fig. 5).

\subsection{REX ISOLDE - A new concept for post-acceleration of radioactive ion beams}

In order to broaden its physics scope and triggered by the successful postacceleration of light RIB at the Louvain-le-Neuve (Belgium) project, ${ }^{16}$ new ways to accelerate the singly charged RIB in a universal, fast, efficient and cost-effective way were explored. This resulted in a novel concept based on ion beam cooling and bunching in the buffer gas of a Penning trap, charge-state breeding in an Electron Beam Ion Source (EBIS) and post-acceleration in a room-temperature linear accelerator. Ion beam cooling and bunching modulates the RIB from ISOLDE into bunches suited for injection in EBIS and was based on the ISOLTRAP experience. The efficient injection of singly charged ions and extraction of highly charged

experimental

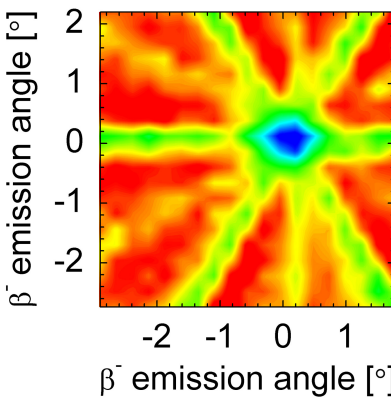

theoretical fit

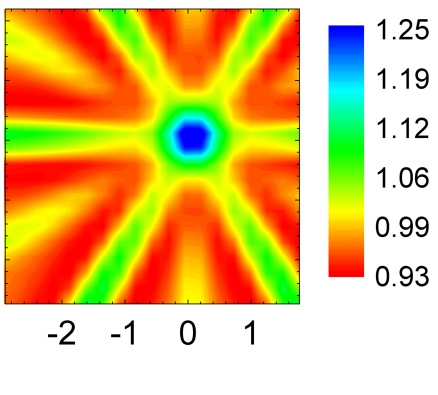

Fig. 5. Emission channeling data recorded using a position sensitive silicon detector obtained after the implantation of ${ }^{56} \mathrm{Mn}$ nuclei into a GaAs (semiconductor) sample. ${ }^{10}$ The detector views the GaAs sample from the implantation site. The colour scale (arbitrary units) corresponds to the angular dependent rate of channeled beta particles emitted from the radioactive ${ }^{56} \mathrm{Mn}$ nuclei sitting in the GaAs matrix. The data (left) are compared to simulations (right) from which the site location of Mn in the GaAs sample can be determined. The latter enhances the understanding of electrical, optical and magnetic influence of dopants in semiconductors. 


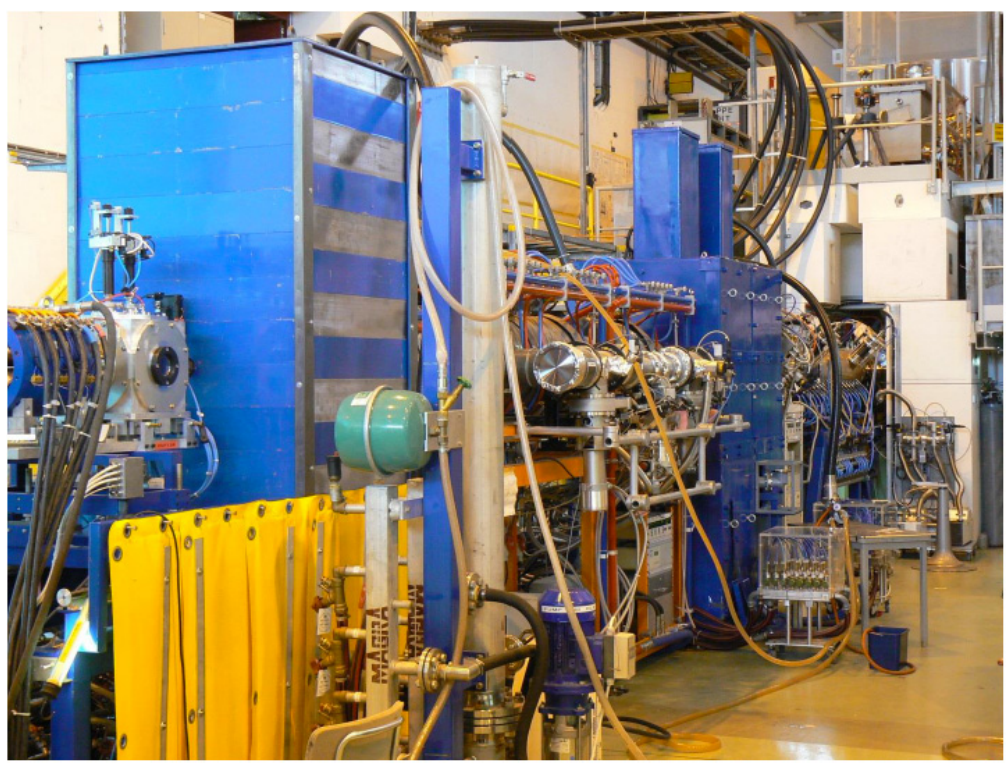

Fig. 6. The REX-ISOLDE post-accelerator delivering radioactive ion beams from ${ }^{6} \mathrm{He}$ to ${ }^{224} \mathrm{Ra}$ with energies up to $3 \mathrm{MeV} / \mathrm{u}$.

ions from EBIS was based on a concept from the Manne Siegbahn Laboratory (Stockholm, Sweden). Finally, the room temperature accelerator cavities were based on designs from the Max-Planck-Institute for Nuclear Physics (Heidelberg, Germany), the GSI HLI-IH-structure (Darmstadt, Germany) and the lead LINAC at CERN.

While the original goals of this Radioactive beam EXperiment at ISOLDE REX-ISOLDE project ${ }^{17}$ (Fig. 6) were limited to energies up to $2 \mathrm{MeV} / \mathrm{u}$ and masses below $A=50$, the concept proved to be very successful and meanwhile beams with $A / Q$ ratio $<4.5$ and with masses up to 220 have been accelerated up to $3 \mathrm{MeV} / \mathrm{u}$, with efficiencies reaching $10 \%$. Most of the beams have been used for Coulomb excitation measurements or few-nucleon transfer reactions using a dedicated particle and gamma-ray detector array for low-intensity low-multiplicity RIB experiments (see Fig. 7).

\section{Shell Structure: The Decline of the Magic Numbers}

The nucleus presents typical characteristics of few-body and many-body quantum systems at the same time. Its microscopic and mesoscopic manifestation are governed by effective 2- and 3-body interactions of great complexity that depends not only on the distance between nucleons but also on its spins and moments.

In its macroscopic behaviour one observes properties equivalent to those of a liquid drop such as energy surface deformation, vibrations, rotations and shapes. 


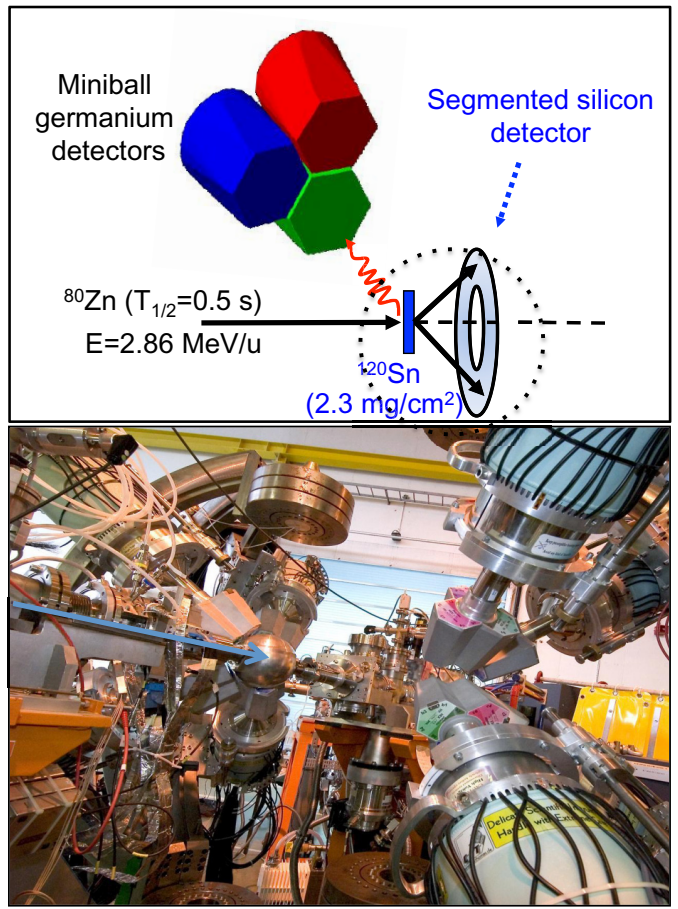

Fig. 7. Top: A schematic drawing of the set-up used for Coulomb excitation experiments. An inelastic collision takes place between a post-accelerated ${ }^{80} \mathrm{Zn}$ beam when hitting a thin ${ }^{120} \mathrm{Sn}$ target. After the collision, the scattered beam and target particles are detected in a segmented silicon detector while the de-exciting gamma rays are recorded in the Miniball germanium array. The beam diagnostics (not shown in the drawing), target and silicon detector array are situated in the spherical reaction chamber. Bottom: The picture shows Miniball germanium array, developed for low-intensity RIB experiments. Eight clusters containing three hexagonal shapes germanium crystals each are positioned around the spherical reaction chamber.

Experimental achievements related with the latter are described in Section 4. Understanding the manifestation of these semiclassical behaviours in terms of the quantum dynamics of the nuclear constituents, protons and neutrons, is one of the main challenges of nuclear theory.

Experiments done with stable and near stable nuclei have shown, that nuclei with $N$ or $Z$ equal to the so-called magic numbers $8,20,28,50,82$ are more difficult to excite than their neighbours. This fact has supported the use of the wellestablished nuclear shell model developed independently by Maria Goeppert Mayer and J. Hans Jensen in 1949 giving them the Nobel Prize in 1963. This model, based on the interactions between nucleons and their arrangement in orbits, has been a success for the understanding of nuclear properties for stable or near stable atomic nuclei. The nuclei with magic numbers exhibit highly symmetric spherical configurations and are since then the milestones of the nuclear landscape. Although this model emerged from a pure phenomenological approach, modern nuclear 
theory can trace the magic numbers down to nucleon-nucleon forces derived from low-energy QCD.

The advent of experimental facilities for the production of radioactive nuclei permitted to reach nuclei with a completely different balance between protons and neutrons, i.e. different isospin. It turned out that the traditional shell structure changed in some regions of the nuclear chart, showing dramatic effects on the neutron-rich side near the neutron binding limit, the neutron drip-line, where the magic character appears at different, $N$ or $Z$ values. Many current studies of nuclear structure with exotic radioactive nuclei focus on the question of whether these magic numbers persist or are altered in going away from the 'valley of stability'. These studies challenge the predictive power of nuclear theory and will eventually lead the way towards a universal description of nuclear structure.

In this section we will describe CERN's contribution to the discovery of the decline of the classic magic numbers. This discovery has changed the perception of nuclear systems and questioned established knowledge. Although the first anomaly in the expected order of nuclear orbits was observed in 1960 in ${ }^{11} \mathrm{Be},{ }^{18}$ it was the measurement of the masses of the exotic sodium isotopes, ${ }^{31,32} \mathrm{Na}$ performed at CERN PS that revealed that these nuclei were tighter bound than expected. ${ }^{19}$ The excess of binding energy was associated with deformation and explained due to the excitation of a neutron across the $N=20$ gap. This was soon further supported by measurements of other ground-state properties: spin, magnetic moments and mean charged radii. ${ }^{20}$ Further, the sodium beta-decay studies allowed for the determination of the first excited states of the magnesium isobars, ${ }^{21}$ while the highly sensitive collinear laser techniques allowed for the mapping of the ground state properties of the neutron rich magnesium isotopes. ${ }^{22}$ The data revealed that the $N=20$ isotopes undergo a sudden onset of deformation and thus the expected magicity vanishes. The shell gap in these neutron-rich isotopes is not robust enough to avoid excitations to a higher shell. This gives rise to quadrupole correlations that favour deformation. A schematic representation of this effect of inversion of orbits is shown in Fig. 8. Many studies have been dedicated to map and define the so-called island of inversion region where ${ }^{32} \mathrm{Mg}$ is situated in the centre. The ${ }^{30} \mathrm{Mg}(N=18)$ isotope has a spherical $0^{+}$ground state with an excited $0^{+}$state at $1788.2 \mathrm{keV}$. Based on the measured monopole strength it could be shown within a two-level model approach that the $0_{2}^{+}$state is strongly deformed. ${ }^{23}$ Moreover theory predicts that its wave function contains a strong intruder configuration, i.e. shape coexistence (see next section). The ground-state of ${ }^{32} \mathrm{Mg}$, a semi-magic nucleus with $N=20$, is strongly deformed as shown from the large $\mathrm{B}\left(\mathrm{E} 2 ; 0_{g s}^{+} \rightarrow 2_{1}^{+}\right)$value. Thus it appears that in ${ }^{32} \mathrm{Mg}$ an inversion takes place: the ground state being deformed and composed of intruder configurations, while a so far unidentified excited $0^{+}$state being spherical. The best proof would be to identify this near-spherical excited $0^{+}$ state, the analogue of the $0^{+}$ground state in ${ }^{30} \mathrm{Mg}$ and to characterise the underlying neutron particle-hole structure of the ground and excited $0^{+}$states. In spite of several attempts this state could not be identified before the work at ISOLDE. 

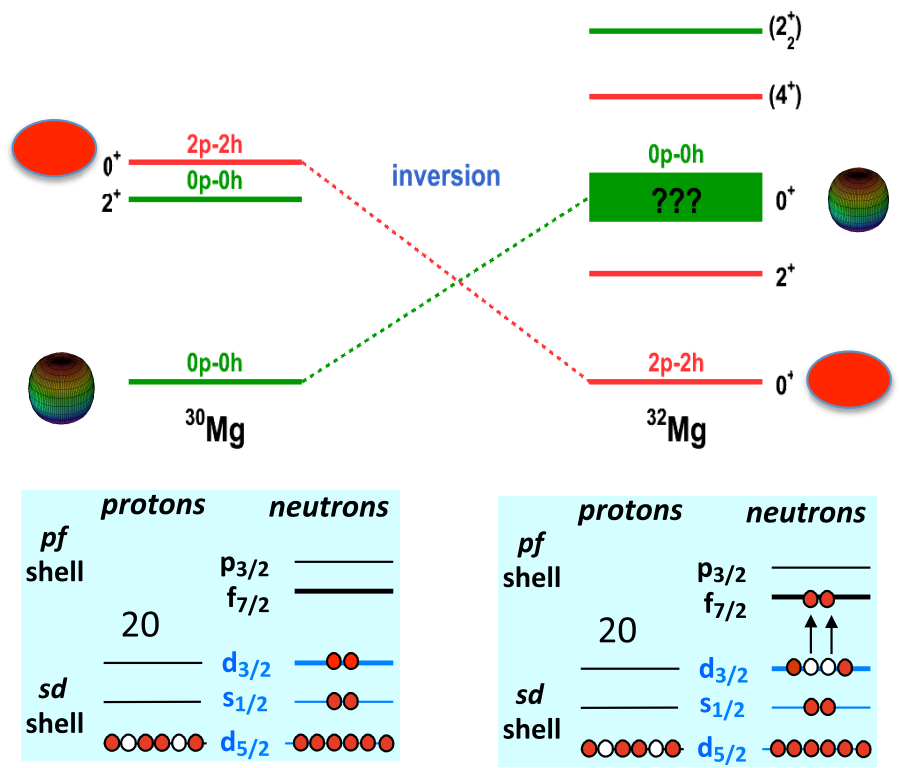

Fig. 8. The fascinating phenomenon of different nuclear shapes coexisting at similar energies the difference is less than $1 \%$ of the total binding energy. It is the reduction of the $N=20$ shell gap that enables neutron-pair excitations across the $N=20$ shell gap leading to quadrupole correlations and giving rise to low-lying deformed two-particle two-hole $2 p-2 h$ states. The so-called intruder states with two neutrons in the $p f$ shell (right: ${ }^{32} \mathrm{Mg}$ ground state) coexist at low excitation energy with the normal spherical zero-particle zero-hole $(0 p-0 h)$ neutron states in the $s d$ shell (left: ${ }^{30} \mathrm{Mg}$ ground state).

A key idea was to study the addition of two neutrons to the spherical ground state of ${ }^{30} \mathrm{Mg}$ populating either the deformed ground state in ${ }^{32} \mathrm{Mg}$ or the excited presumed spherical $0^{+}$state in ${ }^{32} \mathrm{Mg}$. The proof for the correctness of this assumption was achieved at ISOLDE when a $1.8 \mathrm{MeV} / \mathrm{u}$ beam of ${ }^{30} \mathrm{Mg}$ $\left(T_{1 / 2}=335 \mathrm{~ms}\right)$ was used to populate the ground-state and excited states in ${ }^{32} \mathrm{Mg}$ by a two-neutron $(t, p)$ transfer reaction in inverse kinematics, so called when the heavy element is the projectile. The detection of protons and gamma rays were done with the T-REX array and the MINIBALL Ge detector array, see Fig. 7. The T-REX is a $4 \pi$ array consisting of a barrel of silicon-strip detectors together with an annular double-sided segmented silicon-strip detector of CD shape. Energy and direction of emission were measured for protons, deuterons and tritons. By studying angular distributions for the emitted protons, one can determine the angular momentum transferred and from that the spin and parities of the states populated can be deduced. In the experiment, an excited $0^{+}$state at $1058 \mathrm{keV}$ was identified that appears to be an excellent candidate for the spherical state shape co-existing with the strongly deformed $0^{+}$ground state. ${ }^{24}$ From the $(t, p)$ crosssections neutron occupancies across the $N=20$ shell gap for the states in ${ }^{32} \mathrm{Mg}$ were inferred, confirming the inversion and occurrence of shape coexistence. 
The disappearance of magic numbers far from stability is accompanied by the emergence of new ones. Its proper prediction is closely related to our understanding of the different components of the strong force that acts between protons and neutrons. Very recently the predicted magic number far from stability, $N=32$, was confirmed in the study of calcium $(Z=20)$ isotopes at the verge of existence. The ${ }^{51-52} \mathrm{Ca}$ masses were determined using the ISOLTRAP Penning-trap mass spectrometer described in Section 6 and for the extremely rarely produced and short-lived species, ${ }^{53-54} \mathrm{Ca}$, a multi-reflection time-of-flight spectrometer was used. The latter was designed for isobar separation and used for the first time for mass determination. The measured masses confirmed the existence of a prominent shell closure at $N=32$ and provided a formidable benchmark for nuclear theory. ${ }^{25}$

\section{Nuclear Shapes — Shape Coexistence and Quadrupole Deformations}

Atomic nuclei exhibit single-particle and collective degrees of freedom. Understanding the delicate balance between these two extremes underpinning the structure of atomic nuclei is a challenge for theory. In general single-particle effects dominate the structure of nuclei at and around closed proton and neutron shells, while deformation is observed in nuclei situated on the nuclear chart in between doubly closed shell nuclei. Shapes have been studied at ISOLDE using laser spectroscopy and Coulomb excitation measurements. While the former method results in charge radii, magnetic dipole and electrical quadrupole moments of ground states and longlived isomeric states, the latter allows for the determination of quadrupole moments and quadrupole or higher order transition strengths of excited states. Throughout the nuclear chart experimental evidence has been accumulated for a phenomenon called shape coexistence whereby quantum states with different deformation but similar binding energy appear at low energies in the nucleus. In the heavy nuclei shape coexistence was discovered at ISOLDE serendipitously in the light mercury isotopes in optical spectroscopy measurements. ${ }^{14}$ The strong staggering in the charge radii for the lightest mercury isotopes (see Fig. 9) was interpreted as due to the appearance in the odd-mass mercury isotopes of a strongly deformed ground state co-existing with a more spherical isomer. The in-source laser spectroscopy data obtained in the neighbouring lead and polonium isotopes ${ }^{26,28}$ combined with Coulomb excitation measurements in this region using the post-accelerated REX beams allowed to deduce the oblate nature of the ground-state deformation and supported the proposed interpretation of shape coexistence induced by particlehole excitation across the closed proton shell.

The prevalent shape of nuclei is quadrupole deformation, symmetric against reflection, but in some heavy unstable nuclei circumstantial evidence for octupole deformation has been reported. This type of reflection-asymmetric or pear shaped deformation is not only important to test nuclear models, but isotopes exhibiting 


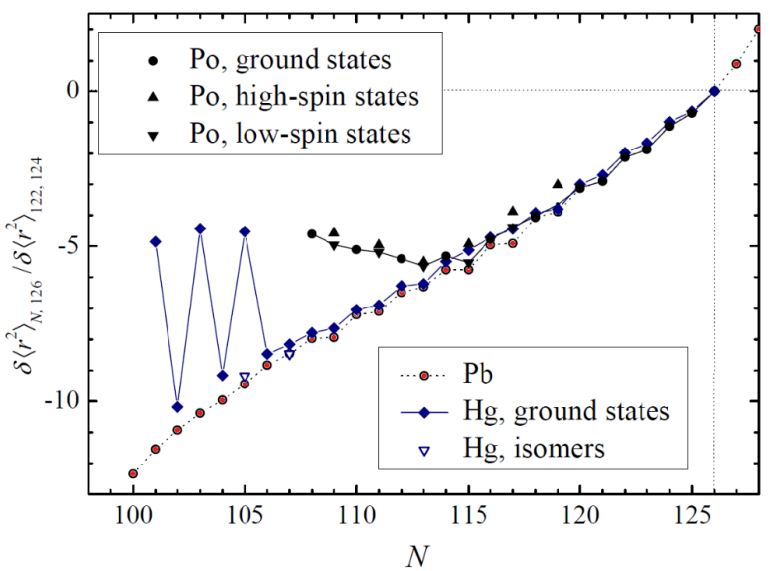

Fig. 9. Relative change in mean-square nuclear charge radii, $\delta\left\langle r^{2}\right\rangle$, for the even- $Z{ }_{80} \mathrm{Hg}$ (blue), ${ }_{82} \mathrm{~Pb}$ (red) and ${ }_{84} \mathrm{Po}$ (black) isotopes. While the relative change in charge radii of one isotope compared to its neighbour for the heaviest isotopes are very similar for these three elements, large differences are observed further away from the $N=126$ neutron shell closure. The large staggering observed in the $\mathrm{Hg}$ data is interpreted as shape coexistence caused by the occupation of specific single-particle states. The deviation observed for the Po isotopes is linked to an onset of collective behaviour possibly caused by the same mechanism. Adapted from Ref. 26.

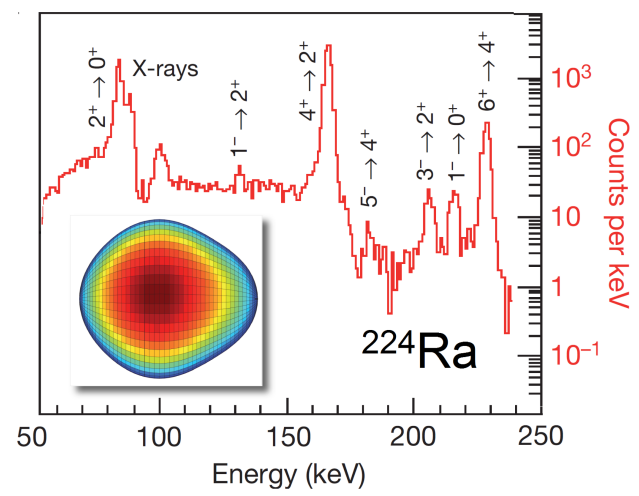

Fig. 10. Part of the gamma-ray energy spectrum obtained after Coulomb excitation of ${ }^{224}$ Ra. From the intensities of the gamma-rays (indicated with the spin and parity of initial and final state), especially the ones de-exciting negative parity states, information on the octupole transition strength was deduced evidencing enhanced octupole deformation. ${ }^{27}$ The inset shows the static octupole deformation of ${ }^{224} \mathrm{Ra}$ in the intrinsic frame as deduced from the experiment.

this type of deformation are ideal probes to look for physics beyond the Standard Model. For the search of an atomic electric-dipole moment in odd-mass isotopes, static electric octupole deformation of the atomic nucleus amplifies the sensitivity by several orders of magnitude. In a recent Coulomb excitation experiment using energetic beams of ${ }^{224} \mathrm{Ra}$, enhanced octupole deformation was evidenced through the measurement of octupole transition strengths (see Fig. 10). ${ }^{27}$ This 
constrains the region of suitable isotopes for studies of the atomic electric-dipole moments.

\section{Nuclear Halos}

A considerable fraction of the experimental programme at the ISOLDE Facility concerns studies of beta decay, which is a well-proven probe of nuclear structure as well as of weak interactions (see Section 6). There is an important difference between beta-decay of near stable nuclei and those in the drip-line regions. Close to stability, the transitions occur between discrete bound levels while the decay closer to the drip-lines also involves states in the continuum. In the neighbourhood of the drip-lines one also encounters beta-delayed particle emission processes, i.e. the particle emission, mediated by the strong force, is delayed as the de-exciting state is slowly populated in the beta decay process. In near drip-line nuclei this decay mode dominates over decays to bound states. ${ }^{29}$ A quite spectacular example of a beta-delayed particle emitter nucleus is provided by the last particle-bound lithium isotope, ${ }^{11} \mathrm{Li}$. This nucleus has a beta-decay $Q$ value, i.e. the mass difference between mother and daughter nuclei, of $20.623 \mathrm{MeV}$, while the neutron separation energy of its daughter, ${ }^{11} \mathrm{Be}$, is as low as $504 \mathrm{keV}$. This energy unbalance opens up several possible beta-delayed particle emission channels, as illustrated in Fig. 11. In a
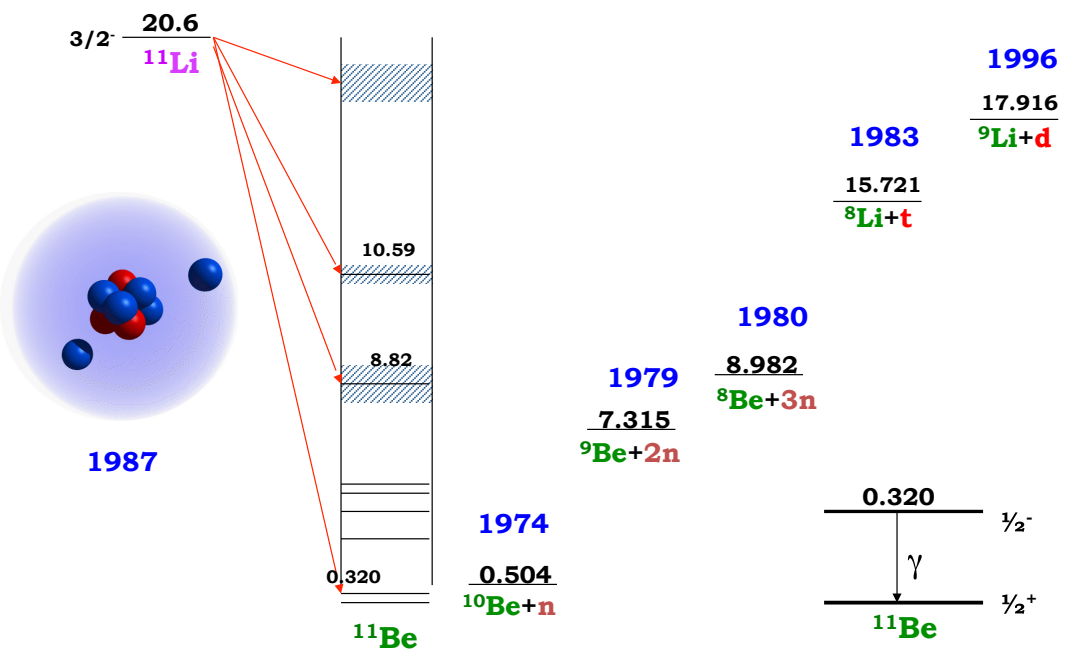

1980

1979

7.315

$\frac{7 \mathrm{Be}+2 \mathrm{n}}{2}$

\subsection{2}

${ }^{8} \mathrm{Be}+3 \mathrm{n}$

Fig. 11. The two-neutron halo nucleus ${ }^{11} \mathrm{Li}$. The very high $Q$-value of $20.6 \mathrm{MeV}$ for beta-decay (red arrows) combined with the comparably low separation energies for various particles in the daughter nucleus ${ }^{11} \mathrm{Be}$ results in a multitude of different beta-delayed particle emission decay modes in this nucleus. The ${ }^{11} \mathrm{Be}$ excitation energies and threshold energies for the different betadelayed decay modes in $\mathrm{MeV}$ (in black) are given relative to the ${ }^{11}$ Be ground state. The years (in blue) are given when these decay modes were first observed and when the halo structure of ${ }^{11} \mathrm{Li}$ was suggested. 
number of experiments at ISOLDE at the end of the seventies and the beginning of eighties the decay modes of beta-delayed two-neutron, three-neutron and triton emission were observed for the first time.

Just at the time when the study of ${ }^{11} \mathrm{Li}$ was in focus at ISOLDE, a group at Berkeley led by I. Tanihata ${ }^{30}$ studied interaction cross-sections of Li isotopes. The remarkable result of this experiment was a large and abrupt increase in the matter radius for ${ }^{11} \mathrm{Li}$. Scientists working with ${ }^{11} \mathrm{Li}$ at ISOLDE came with an explanation for the increase of the ${ }^{11} \mathrm{Li}$ radius based on the low two-neutron separation energy. ${ }^{31}$ A model, where ${ }^{11} \mathrm{Li}$ should have a novel type of structure, a halo, was proposed. The basic idea is that the ${ }^{11} \mathrm{Li}$ nucleus is built up by a ${ }^{9} \mathrm{Li}$ core surrounded by two loosely bound neutrons forming a veil of neutron matter around the core.

The realisation of the occurrence of halo structure at the drip-lines sparked off an intense experimental activity and many nuclei having neutron and proton halos are known today. ${ }^{32}$ Important early ingredients for the understanding of the halo structure came from ISOLDE where the spin, magnetic moment and the electric quadrupole moments were measured for the chain of bound $\mathrm{Li}$ isotopes in a combination of optical and beta-decay measurements, ${ }^{33,}{ }^{34}$ results that later got confirmed and improved. ${ }^{35}$ The results showed that the magnetic dipole and the electric quadrupole moments of the two isotopes ${ }^{9} \mathrm{Li}$ and ${ }^{11} \mathrm{Li}$ were very similar. This proves that the increase in the radius arises from the neutron tail, while the charged core is little affected.

Another consequence of the two-neutron halo structure is the occurrence of beta-delayed deuteron emission. The $Q$ value for this process is $Q\left(\beta^{-} \mathrm{d}\right)=(3.007-$ $\left.\mathrm{S}_{2 n}\right) \mathrm{MeV},{ }^{36}$ where $\mathrm{S}_{2 n}$ is the separation energy for the last two neutrons. The occurrence of this decay mode was shown at ISOLDE for the first time for the two-neutron halo nucleus ${ }^{6} \mathrm{He}^{37}$ and later also for ${ }^{11} \mathrm{Li}^{38}$

The beta-decay daughter of ${ }^{11} \mathrm{Li}$ is ${ }^{11} \mathrm{Be}$, which is an example of a oneneutron halo nucleus. Its magnetic moment was measured at ISOLDE in a very beautiful experiment. ${ }^{39}$ The Be isotopes are produced in a $\mathrm{UC}_{2}$ target matrix irradiated by $1 \mathrm{GeV}$ protons from the PS Booster (see Section 2.1). The produced Be evaporates into a tungsten cavity, where two laser beams excite the atoms from the $2 s^{2}{ }^{1} S_{0}$ atomic ground state to an auto-ionising state via the atomic $2 s 2 p^{1} P_{1}$ state. The nuclei of the ${ }^{11} \mathrm{Be}^{+}$beam are then optically polarised by a collinear frequency-doubled $\mathrm{CW}$ dye laser beam. The polarised ions are implanted in a Be crystal placed in the centre of an NMR magnet. The first-forbidden beta decay to ${ }^{11} \mathrm{~B}$ of the polarised nuclei are detected with two scintillators and the beta-decay asymmetry is measured. From the observed Larmour frequency the magnetic moment is determined as $\mu\left({ }^{11} \mathrm{Be}\right)=-1.6816(8) \mu_{N}$. This value is confirming a $16 \%$ core polarisation admixture in the ${ }^{11} \mathrm{Be}$ ground-state wave function. ${ }^{40}$

A major experimental success was the use of the collinear laser technique to determine the nuclear charge radii for Be isotopes. In collinear laser spectroscopy 


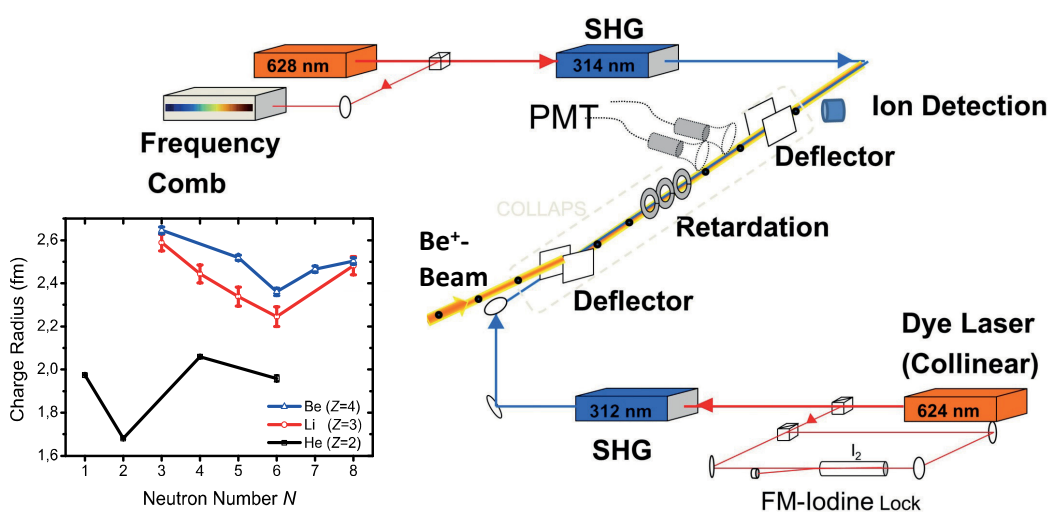

Fig. 12. Set-up for collinear laser spectroscopy with parallel and antiparallel excitation and a frequency comb as reference for the determination of the charge radius for Be isotopes. Key components of the experimental set-up are shown (SHG: second harmonic generator, PMT: photomultiplier tube). The inset shows the state-of-the-art of charge radii measurements ${ }^{41}$ for light drip-line nuclei.

the laser beam is superimposed with a beam of fast (typically $30-60 \mathrm{keV}$ ) ions or atoms and the resonance fluorescence is detected with a photomultiplier perpendicular to the flight direction. Since the atoms are propagating in a parallel or antiparallel manner to the laser beam, the resonance frequency of the atom is shifted in the laboratory system by the relativistic Doppler effect. ${ }^{42}$ Collinear laser spectroscopy has the advantage that the acceleration of an ion ensemble with a static electric potential compresses the longitudinal velocity distribution. Thus, the Doppler width is considerably reduced. With a beam of $\mathrm{Be}^{+}$ions, a frequency comb and measuring the absolute transition frequencies for parallel and antiparallel geometry of the ion and laser beams, see Fig. 12, a hitherto impossible precision was obtained. The beauty of the technique is that the rest frame frequency, $\nu_{0}$, is obtained independent of the acceleration voltage by combining the measured absolute transition frequencies for parallel $\left(\nu_{p}\right)$ and antiparallel $\left(\nu_{a}\right)$ laser beams so that $\nu_{p} \nu_{a}=\nu_{0}^{2} \gamma^{2}(1+\beta)(1-\beta)=\nu_{0}^{2}$. The required accuracy in the isotope shift measurement of $1 \mathrm{MHz}$ was obtained. The charge radii were observed to decrease for the isotopes ${ }^{7} \mathrm{Be}$ to ${ }^{10} \mathrm{Be}$ and then increase for ${ }^{11} \mathrm{Be}$, see Fig. 12. ${ }^{43}$ This increase of the charge radius is expected since the centre of mass and the centre of charge do not coincide in a one-neutron halo nucleus like ${ }^{11} \mathrm{Be}$.

\section{Fundamental Interaction Studies}

Radioactive beams are ideal probes for fundamental studies of weak interaction and of the Standard Model (SM) in general. Among others, precision measurements of masses, half-lives and branching ratios of superallowed $\beta$-emitters allow in 
combination with nuclear theory a precise determination of the first $\left(V_{u d}\right)$ element of the Cabibbo-Kobayashi-Maskawa (CKM) quark-mixing matrix, which relates the quark weak-interaction eigenstates to the quark mass eigenstates assuming three quark generations. Taking $V_{u s}$ and $V_{u b}$ from the Particle Physics Data Group (PDG), a stringent top-row unitarity test of the CKM matrix can be performed: ${ }^{44}$

$$
\sum_{j}\left|V_{u j}\right|^{2}=\left|V_{u d}\right|^{2}+\left|V_{u s}\right|^{2}+\left|V_{u b}\right|^{2}=1 .
$$

Any deviation from 1 can be related to concepts beyond the SM such as the existence of an additional $Z$-boson or the existence of right-handed currents in the weak interaction. $V_{u d}$ can be determined from the fundamental vector coupling constant $G_{V}$ and the well-known weak-interaction constant $G_{F}$ of purely leptonic muon decay: $V_{u d}=G_{V} / G_{F}$, where $G_{V}$ in turns can be derived from the corrected strengths ( $F t$-value) of superallowed $\beta$-transitions, which are a function of the experimental parameters: $\beta$-decay $Q$-value, half-life $T_{1 / 2}$, and branching ratio $b$, as well as of different correction terms including isospin-symmetry-breaking and radiative correction. The uncorrected $f t$-values can be derived purely from nuclear physics experiments, namely from mass, half-life and branching ratio measurements of superallowed $\beta$-transitions. Many ISOLDE experiments, especially highprecision mass measurements with the Penning-trap mass spectrometer ISOLTRAP (see Fig. 13), being the first of its type installed at a radioactive ion beam facility in 1986, have contributed to this kind of research, providing the most accurate $V_{u d}$ value to date of $\left|V_{u d}\right|=0.97417(21) .{ }^{44}$ Taking $V_{u s}$ and $V_{u b}$ from PDG, one obtains the result:

$$
\left|V_{u d}\right|^{2}+\left|V_{u s}\right|^{2}+\left|V_{u b}\right|^{2}=0.99978(55),
$$

i.e. the unitarity is fully satisfied to a precision of $0.06 \%$.

Another pillar of the SM is the conserved-vector-current (CVC) hypothesis, stating that the vector part of the weak interaction is not influenced by the strong interaction. ${ }^{44}$ Thus, $F t$ should be constant for all superallowed transitions. Taking all presently available data result in an amazing consistency at the $0.03 \%$ precision level. ${ }^{44}$

Radioactive nuclei are also ideal systems to put constraints on scalar currents in weak interaction which is supposed to be a pure A-V interaction. One highlight example of ISOLDE is the measurements on ${ }^{32} \mathrm{Ar}\left(T_{1 / 2}=98 \mathrm{~ms}\right)$ an isotopic spin $T_{z}=-2$ nucleus, which was discovered at ISOLDE in $1977 .{ }^{45}$ One of the best known values for the positron-neutrino angular correlation coefficient $a$ was determined in the $0^{+} \rightarrow 0^{+} \beta$-decay of the ${ }^{32} \mathrm{Ar}$ experiment by Adelberger et al. in 1999. ${ }^{46}$ The effect of lepton recoil on the shape of the narrow proton peak in the particle spectrum following the superallowed decay was analysed. Since the mass of ${ }^{32} \mathrm{Ar}$ was only known to an uncertainty of $50 \mathrm{keV}$ at that time, the accuracy in the determination of $a$ was limited to $6 \%$. Thus, the mass prediction by the isobaric multiplet mass equation (IMME) was used instead and 


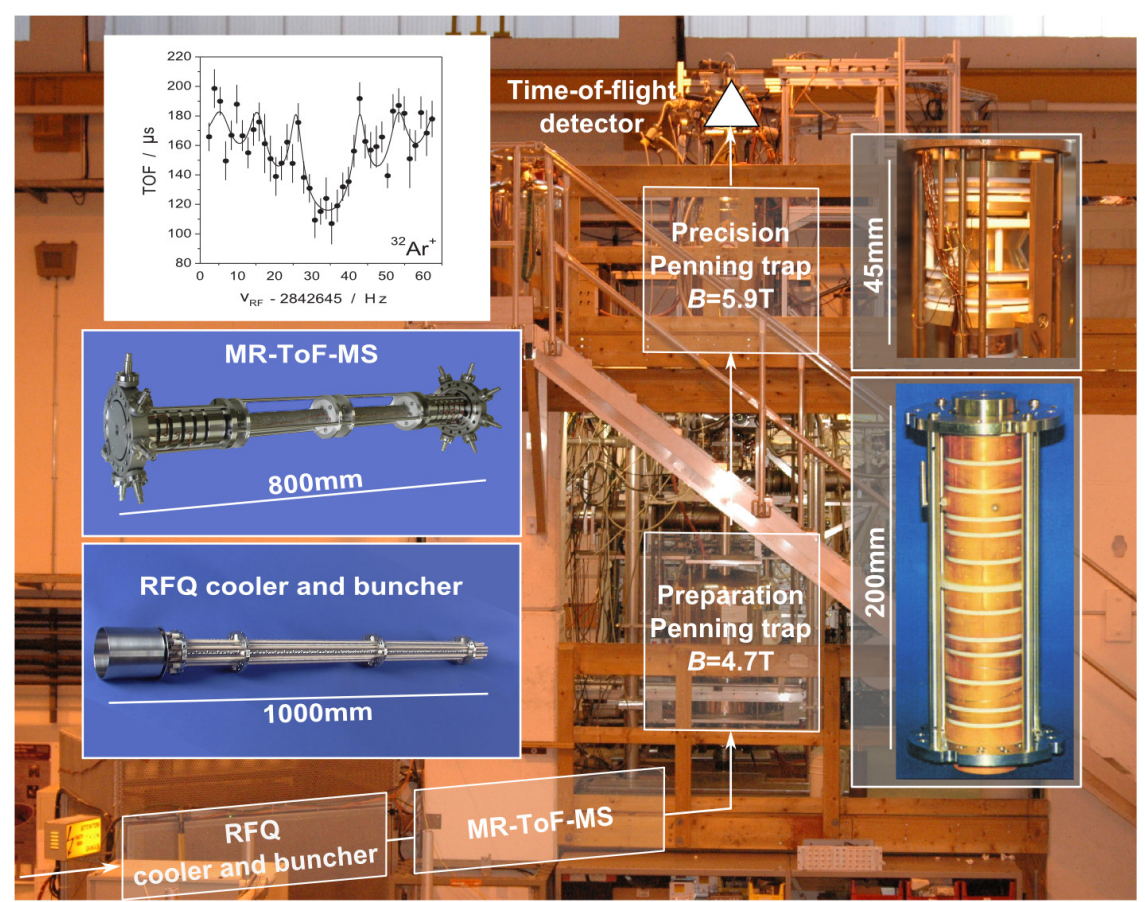

Fig. 13. Present set-up of the ISOLTRAP Penning-trap mass spectrometer at ISOLDE for high-precision mass measurements on short-lived nuclides. The inset shows on the top left the cyclotron resonance of ${ }^{32} \mathrm{Ar}^{+}$with the fit of the theoretically expected line-shape. The other insets show the different trapping devices at ISOLTRAP. At the time of the Ar measurement in 2001 the multi-reflection time-of-flight (MR-ToF-MS) spectrometer was not installed at ISOLTRAP.

resulted in the beta-neutrino correlation coefficient for vanishing Fierz interference of $a=0.9989 \pm 0.0052$ (stat) \pm 0.0039 (syst) at the $68 \%$ confidence level, thus being fully consistent with the SM prediction. Furthermore, a new limit on the masses of scalar particles with gauge coupling strength could be derived being at that time $M_{S} \geq 4.1 M_{W}$. A few years later the mass of ${ }^{32} \mathrm{Ar}$ was measured for the first time directly by Penning-trap mass spectrometry at ISOLTRAP ${ }^{47}$ (see inset of Fig. 13) with a mass uncertainty of only $\delta m=1.8 \mathrm{keV} / c^{2}$, thus allowing for an improved value of the positron-neutrino angular correlation coefficient $a$ and relying no longer on the IMME prediction.

\section{ISOLDE at the Doorstep to the Next Half-Century}

In the period of writing this historic review of some of the landmark experiments, we witness an intense period building up new projects for the future:

The new post-accelerator structure HIE-ISOLDE. The HIE-ISOLDE (High Intensity and Energy) project will provide major improvements in energy range, 
beam intensity and beam quality. An important element of the project will be an increase of the final energy of the post-accelerated beams to $10 \mathrm{MeV} / \mathrm{u}$ throughout the periodic table. The first stage will boost the energy of the current REX-LINAC to $5.5 \mathrm{MeV} / \mathrm{u}$ where the multistep Coulomb excitation cross-sections are strongly increased with respect to the previous $3 \mathrm{MeV} / \mathrm{u}$ and many transfer reaction channels will be opened. ${ }^{48}$ The construction of the Linac is underway and the full physics programme with post accelerated beams up to $5.5 \mathrm{MeV} / \mathrm{u}$ will start in 2016 .

The TSR storage ring. Recently, a low-energy storage ring was proposed to be installed at HIE-ISOLDE using the existing ring TSR presently in operation at the Max-Planck-Institute for Nuclear Physics in Heidelberg. ${ }^{49}$ The addition of a storage ring to an ISOL facility opens up an extremely rich scientific programme in nuclear physics, nuclear astrophysics and atomic physics. Reaction and decay studies can benefit from the "recycling" of the rare exotic nuclei stored in the ring and from low background conditions. Studies of the evolution of the atomic structure can be extended to isotopes outside the valley of stability. In addition to experiments performed using beams recirculating within the ring, cooled beams can be extracted and exploited by external spectrometers for high-precision measurements.

The CERN-MEDICIS project. Building further on ISOLDE's know-how in RIB production and on CERN's proton beam capabilities, the CERN-MEDICIS (Medical Isotopes Collected from ISOLDE) project was initiated. MEDICIS will exploit targets installed at ISOLDEs beam dump position and produce long-lived radioisotopes for fundamental studies in cancer research, for new imaging and therapy protocols and for pre-clinical trials.

These projects will create new opportunities for radioactive beam research and bring ISOLDE at the doorstep of the next half-century.

\section{Acknowledgments}

It has been a privilege for us to write this chapter in the book celebrating CERN's 60 years. ISOLDE has been around at CERN for 50 years and we want to take this opportunity to thank, on behalf of all our experimental and theoretical colleagues that have worked at ISOLDE, CERN for its generous support and especially the ISOLDE technical team has always been supporting the users and made this story possible. We also want to convey our sincere thanks to the national funding bodies for their continued support, which made this 50 years journey possible.

\section{References}

1. O. Kofoed-Hansen and K. O. Nielsen, Phys. Rev. 82, 96 (1951); Kgl. Dan. Vidensk. Selsk. Mat. Fys. Medd. 26, No. 7 (1951).

2. M. J. G. Borge et al., Phys. Lett. B 317, 25 (1993). 
3. H. L. Ravn and B. W. Allardyce, On-line Mass separators, in Treatise on Heavy Ion Science, Vol. 8 (Springer, 1989), pp. 363-439.

4. P. Van Duppen and K. Riisager, J. Phys. G 38, 1 (2011).

5. H.-J. Kluge et al., Laser Ion Sources, in Proc. Accelerated Radioactive Beams Workshop, Parksville, Canada (1985).

6. F. Scheerer et al., Rev. Sci. Instr 63, 2831 (1992).

7. V. Mishin et al., NIM B 73, 550 (1993).

8. K. Blaum et al., NIM B 204, 331 (2003).

9. D. Fink et al., NIM B 344, 83 (2015); NIM B 317, 661 (2013).

10. L. M. C. Pereira et al., Appl. Phys. Lett. 98, 201905 (2011).

11. U. Köster et al., NIM B 160, 528 (2000).

12. J. Van Roosbroeck et al., Phys. Rev. Lett. 92, 112501 (2004).

13. I. Stefanescu et al., Phys. Rev. Lett. 98, 122701 (2007).

14. J. Bonn et al., Phys. Lett. B 38, 308 (1972).

15. S. Rothe et al., Nature Comm. 4, 1835 (2013).

16. P. Delrock et al., Phys. Rev. 67, 808 (1991).

17. O. Kester et al., NIM B 204, 20 (2003).

18. I. Talmi and I. Unna, Phys. Rev. Lett. 4, 469 (2006).

19. C. Thibault et al., Phys. Rev. C 12, 644 (1975).

20. G. Huber et al., Phys. Rev. C 18, 2342 (1978).

21. D. Guillemaud-Mueller et al., Nucl. Phys. A 426, 37 (1984).

22. G. Neyens et al., Phys. Rev. Lett. 94, 022501 (2005).

23. W. Schwerdtfeger et al., Phys. Rev. Lett. 103, 012501 (2009).

24. K. Wimmer et al., Phys. Rev. Lett. 105, 252501 (2010).

25. F. Wienholtz et al., Nature 498, 346 (2013).

26. M. D. Seliverstov et al., Phys. Lett. B 719, 362 (2013).

27. L. Gaffney et al., Nature 497, 199 (2013).

28. H. De Witte et al., Phys. Rev. Lett. 98, 112502 (2007).

29. M. J. G. Borge, Phys. Scr. T 152, 014013 (2013).

30. I. Tanihata et al., Phys. Rev. Lett. 55, 2676 (1985).

31. P. G. Hansen and B. Jonson, Europhys. Lett. 4, 409 (1987).

32. K. Riisager, Phys. Scr. T 152, 014001 (2013).

33. E. Arnold et al., Phys. Lett. B 197, 311 (1987).

34. E. Arnold et al., Phys. Lett. B 281, 16 (1992).

35. R. Neugart et al., Phys. Rev. Lett. 101, 132502 (2008).

36. B. Jonson and K. Riisager, Nucl. Phys. A 693, 77 (2001).

37. K. Riisager et al., Phys. Lett. B 235, 30 (1990).

38. I. Mukha et al., Phys. Lett. B 367, 65 (1996).

39. W. Geitner et al., Phys. Rev. Lett. 83, 3792 (1999).

40. T. Suzuki, T. Otsuka and A. Muta, Phys. Lett. B 364, 69 (1995).

41. K. Blaum, J. Dilling and W. Nörtershäuser, Phys. Scr. T 152, 014017 (2013).

42. S. L. Kaufman, Opt. Commun. 17, 309 (1976).

43. W. Nörtershäuser et al., Phys. Rev. Lett. 102, 062503 (2009).

44. J. C. Hardy and I. S. Towner, Phys. Rev. C 91, 025501 (2015).

45. E. Hagberg et al., Phys. Rev. Lett. 39, 792 (1977). 
46. E. G. Adelberger et al., Phys. Rev. Lett. 83, 1299 (1999) and E. G. Adelberger et al., Phys. Rev. Lett. 83, 3101 (1999).

47. K. Blaum et al., Phys. Rev. Lett. 91, 260801 (2003).

48. K. Riisager et al. (eds.), HIE-ISOLDE: The scientific opportunities. CERN Report, CERN-006-013.

49. M. Grieser et al., Eur. Phys. J. Special Topics 207, 1 (2012). 